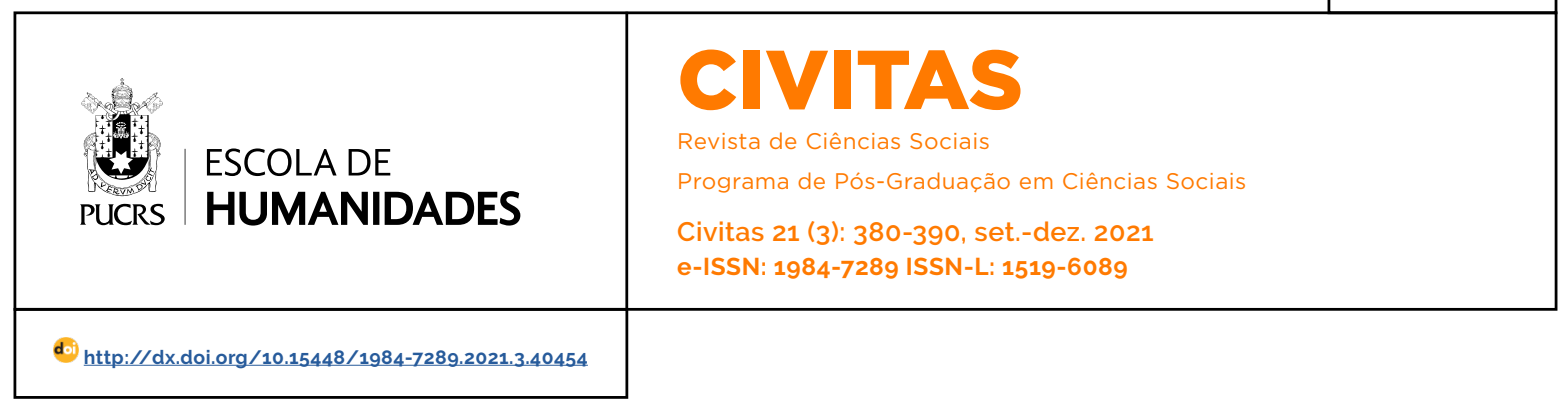

DOSSIÊ: INTERSECCIONALIDADES, DIREITOS E POLITITICAS

\title{
La dimensión global de las políticas públicas de género y salud en América Latina: un análisis decolonial
}

\author{
A dimensão global das políticas públicas de gênero e saúde na América Latina: uma \\ análise decolonial
}

The global dimension of gender and health public policies in Latin America: a decolonial analysis

\section{Laura Cecilia López ${ }^{1}$ orcid.org/0000-0002-2454-063X lauracl@unisinos.br}

\section{Luanda Rejane Soares \\ Sito $^{2}$}

orcid.org/0000-0001-7579-4229 luanda.soares@udea.edu.co

\section{Yadira Eugenia Borrero- Ramirez ${ }^{2}$}

orcid.org/0000-0003-2559-4637 yadira.borrero@udea.edu.co

Recebido em: 23 mar. 2021. Aprovado em: 5 ago. 2021. Publicado em: 8 nov. 2021

\section{(c) (1)}

Artigo está licenciado sob forma de uma licença Creative Commons Atribuição 4.0 Internacional.
Resumen: Se propone un análisis de políticas públicas que articulan género y salud en América Latina, para repensar la dimensión global de la construcción de conceptos, indicadores e instrumentos de evaluación de impactos de estas políticas en la región. Indagamos cómo estudios y evaluaciones globales sobre esas políticas "hacen género" de una manera particular, vinculada a la colonialidad que entreteje las sociedades del Sur Global. La metodología contempló análisis discursivo de publicaciones de dos actores globales destacados en la revisión documental: Cepal y OPS. Entre los resultados, se apuntan diferencias en los modos de "hacer género" de estos dos órganos, con la operación discursiva de supresión de heterogeneidad en la idea de autonomía del primero y con construcciones relacionales de género del segundo vinculadas a la equidad. Se destaca que el alineamiento a políticas neoliberales no permite visualizar instrumentos de justicia social más robustos propuestos en estas evaluaciones. Palabras clave: Género. Colonialidad. Política pública. Salud. Sur global.

Resumo: Propõe-se uma análise das políticas que articulam gênero e saúde na América Latina, a fim de repensar a dimensão global da construção de conceitos, indicadores e instrumentos de avaliação de impactos dessas políticas na região. Indagamos em como os estudos e as avaliações globais sobre essas políticas "fazem gênero" de uma maneira particular, vinculada à colonialidade que atravessa as sociedades do Sul Global. A metodologia contemplou uma análise discursiva de publicações de dois atores globais destacados na revisão documental: a Cepal e a OPS. Entre os resultados, se apontam diferenças nos modos de "fazer gênero" desses órgãos, com a operação discursiva de supressão da heterogeneidade na ideia de autonomia do primeiro e com construções relacionais de gênero do segundo vinculadas à equidade. Destaca-se que o alinhamento a políticas neoliberais não permite visualizar instrumentos de justiça social mais robustos nestas avaliações. Palavras-chave: Gênero. Colonialidade. Política pública. Saúde. Sul global.

Abstract: The purpose of this article is to present an analysis of policies that joint gender and health in Latin America, based on a global dimension of the agenda and the institutionalization of this issues at the region. It focuses on how studies and global assessments of these policies "make gender" precisaly, connected to the coloniality that weaves societies in the Global South. The methodology included a discourse analysis of recent publications provided by two global actors highlighted in the review: Cepal and PAHO. Among the results, there are differences in the of "making gender" of these organs, with the discursive operation of suppression of heterogeneity in the idea of autonomy of the first and with relational constructions of gender of the second linked to equity. It is noteworthy that the alignment to neoliberal policies does not allow us to visualize more robust instruments of social justice proposed in these evaluations. Keywords: Gender. Coloniality. Public Policy. Health. Global South. 


\section{Introducción}

Este articulo propone un análisis de politicas públicas que articulan género y salud en América Latina, para repensar la dimensión global de la construcción de conceptos, indicadores e instrumentos de evaluación de impacto de estas políticas en la región. Se focaliza en cómo los estudios y evaluaciones globales sobre políticas de género y salud "hacen género" de una forma particular, vinculada a la colonialidad que entreteje las sociedades del Sur global. Elegimos el campo sanitario dada la amplia lucha del movimiento feminista por la autonomía del cuerpo $y$. el reconocimiento del rol central del cuidado y su necesaria redistribución.

Partimos de una perspectiva de género relacional, siguiendo a Connell (2015), que piensa cómo las construcciones de femineidades y masculinidades se da en una trama social compleja, considerando procesos históricos constituyentes. La violencia generada por las relaciones coloniales desempeña un papel decisivo en la configuración de sociedades del Sur Global y sus dinámicas de género (Connell 2015). Se entiende la colonización como un acto de dominación estructural y de supresión violenta de la heterogeneidad de los sujetos colonizados (Mohanty 2008). Considerar esta perspectiva conduce a incorporar la dimensión de la colonialidad del género (Lugones 2008) como parte de las consideraciones analíticas.

La teoría relacional propone el género como "un vínculo que implica múltiples personas y categorias, que conecta cuerpos e instituciones" (Connell 2013, 65), e involucra dinámicas sociales, como raza, clase, nacionalidad, posición en el orden mundial. Inversamente, no se puede entender estas categorias sin considerar el género (Connell 2015). Así, la producción de jerarquias raciales, entre cuerpos y el propio binarismo sexual son intrínsecos a la conformación del sistema-mundo colonial/patriarcal/capitalista/ moderno (Lugones 2008).

Según Connell (2013), las relaciones de género tienen una dimensión global, ya que las dinámicas del orden mundial se articulan a dinámicas políticas y económicas y afectan profundamente las vidas localizadas. Entendemos el "hacer género" como una dinámica productora de prácticas sociales e institucionales con efectos de poder sobre los cuerpos y las vidas (Vianna y Lowenkron 2017). Focalizamos aqui el "hacer género" de los actores globales, partiendo del presupuesto que esta construcción tiene efectos potentes en la configuración de nociones que subyacen a la implementación de políticas públicas en los países del Sur Global.

Se realizó una revisión documental que abarcó estudios publicados en las últimas dos décadas con análisis en escala regional de las políticas públicas de género y salud. Entre ellos se construyó un corpus documental con publicaciones de los últimos 5 años (2015-2020) de dos actores globales destacados en la revisión: la Comisión Económica para América Latina y el Caribe - Cepal, y la Organización Panamericana de Salud OPS. Cabe mencionar que, aunque estos estudios hayan sido elaborados por personas concretas, la autoria es de los órganos patrocinadores, produciendo efectos de discurso institucional.

El análisis documental se realizó a través de la comparación de diferencias y similitudes y la expansión de las discusiones en diálogo crítico con los estudios de género y colonialidad. El Análisis Crítico de Discurso guió cómo pensar el carácter productivo del discurso, en doble sentido: cómo el discurso produce la sociedad y la sociedad produce discurso (Berardi 2003). Delineamos como eje analítico la idea de que los actores estatales y globales, al construir conceptos, indicadores e instrumentos para implementar y evaluar las políticas de género, están "haciendo género" en el sentido productivo del discurso, entretejiendo prácticas de dominación y resistencia.

La revisión amplia de estudios de políticas públicas de género y salud de carácter regional nos permite la contextualización de los resultados. Resaltamos principalmente lo que estos estudios muestran sobre la penetración de orientaciones de actores globales en el diseño de politicas públicas de los Estados de la región, sin detenernos en las particularidades de cada país, lo cual necesita otro espacio de debate. 
Los estudios abordan la institucionalización ${ }^{3}$ de las políticas para las mujeres en la región, particularmente la creación de Mecanismos de Adelanto de las Mujeres en el Estado - MAM. Éstos fueron producto de la articulación entre movimientos feministas, actores globales y gobiernos nacionales, en conferencias mundiales y regionales en torno a la igualdad de género (Guzmán y Montaño 2012; Guzmán y Jannotti 2008).

La mayoría de los MAM se establecen a partir de la década de 1980 como órganos dentro de ministerios responsables de políticas sociales de familia, salud y educación. En la década de 1990 se constituyeron como órganos del Poder Ejecutivo concebidos como instancias responsables de la coordinación de políticas de género orientadas por acuerdos internacionales (Guzmán y Montaño 2012). Estos mecanismos, junto con los Planes Nacionales de Igualdad de Género, son considerados por actores globales como parte de las "exigencias del campo de acción política feminista a los Estados, tarea en la que las políticas públicas constituyen una herramienta importante de construcción de nuevas relaciones de igualdad y de fortalecimiento de la democracia" (Cepal 2017, 15).

Sin embargo, situaciones de "vulnerabilidad institucional" afectan a los MAM: recursos insuficientes para implementar agendas, interferencia de "actores de veto" (como la iglesia católica) persiguiendo la agenda de género, como sucede con las demandas de legalización del aborto, educación sexual en escuelas, prevención del Sida, reconocimiento de identidades sexuales plurales (Guzmán y Montaño 2012).

Al analizar las políticas para las mujeres en América Latina entre 1985 y 2010, Guzmán y Montaño $(2012,13)$ resaltan el papel de "ofensiva modernizadora" desde abajo del movimiento feminista, siendo que "la práctica y la teoría feminista pusieron en evidencia el carácter omnipresente del Estado tanto en la construcción y reproducción de las relaciones de género desiguales como en su transformación". En ese sentido, Guzmán y Jan- notti $(2008,113)$ destacan la contribución central de los movimientos feministas a la modernización y democratización de las sociedades latinoamericanas, "insertándose en un campo institucional más amplio de debates y lucha política, donde disputan proyectos de modernización de distintos matices políticos e ideológicos".

Estos estudios consideran condiciones contextuales que marcan el escenario regional "caracterizado por la aceleración de la globalización, la recuperación de los regímenes democráticos y el fortalecimiento de movimientos sociales a nivel nacional e internacional" (Guzmán y Montaño 2012, 11). Sin embargo, no profundizan los drásticos cambios que Latinoamérica sufrió durante las décadas de 1980 y 1990 con la implantación del neoliberalismo a partir de las orientaciones de actores como el Banco Mundial. Las medidas de ajuste estructural y la reducción del Estado tuvieron consecuencias devastadoras, profundizando desigualdades históricas, incrementando las condiciones de precariedad en los sistemas de protección social y reduciendo la política social a asistencialismo focalizado (Basile 2020).

El argumento construido por estos actores para focalizar las políticas fue la "eficiencia" para combatir la pobreza, ya que focalizar a las mujeres, por su rol familiar, por su presencia en asuntos comunales, o por ser mayoría en estratos pobres, impactaría la sociedad como un todo. Esta idea tuvo adherencia regional, principalmente en los programas de transferencia monetaria aplicados en casi toda la región (Fahra 2004). También se puede resaltar el lugar que estas políticas asumieron en el área de salud reproductiva, con la expansión del uso de métodos anticonceptivos centrados en las mujeres (incluyendo métodos quirúrgicos) para la llamada "planificación familiar" propiciada por actores estatales y globales. Estas políticas fueron un intento de regular tanto la reproducción de las mujeres pobres del "Tercer Mundo" por la "alta tasa de natalidad" que se les adjudicaba, como la subsecuente "reproducción

3 La institucionalización es entendida como la legitimación de "expresiones empíricas y materiales de relaciones políticas, prácticas sociales y visiones del mundo" que se transformaron en "cosas públicas y/u oficiales mediante procesos históricos que involucran luchas políticas" (Guzmán y Janotti 2008, 106). 
social de la pobreza" (Del Rio Fortuna 2013); y se las podría interpretar como continuidad de las políticas eugenésicas de "higienización social" que imperaron en la primera mitad del siglo 20 en nuestros países.

La focalización de las políticas se amalgama en cierta medida con las demandas de los movimientos de mujeres, sólo que con sentidos usualmente opuestos y en tensión. Si la propuesta del Banco Mundial apunta a la "eficiencia del gasto" y a una idea "funcionalista" de las mujeres, los movimientos proponen una focalización para ampliar los derechos de ciudadanía, relegados por el Estado (Fahra 2004) y discutir los efectos de las políticas neoliberales en sus vidas (Vargas 2003).

Emergen asuntos criticos en esta "ofensiva modernizadora": si, por un lado, permitió nuevas formas de gestión pública vinculadas a procesos de democracia participativa, por otro, no parece haber abierto una evaluación crítica de la racionalidad neoliberal que permea las políticas y que (re)produce un orden de género desigual. Como expresa Connell (2015), el neoliberalismo se presenta como "neutro" en términos de género, promoviendo al individuo (sin género) como "actor racional" y "emprendedor". Sin embargo, éste funciona como una forma de politica masculinista debido al poderoso papel que desempeña el Estado en el orden de género.

Para Tajer (2012,15), los hombres son los grandes ausentes de las políticas focalizadas en las mujeres y la expectativa recae sobre su rol de "proveedores económicos y sociales, siendo sancionados socialmente cuando no pueden o quieren cumplir con dicho rol". Esta ausencia refuerza la expectativa social de que las mujeres continúen siendo "las principales cuidadoras de la familia y las agentes del sistema de salud en los hogares basándose en su capacidad empática, receptiva y comprensiva".

Otro destaque es que las políticas focalizadas en las mujeres reproducen una "mujer genérica", aislándola no sólo de las dinámicas de género (al no cuestionarse las masculinidades producidas), sino también de otras dinámicas interseccionadas: clase, raza, disidencias sexuales. Mohanty (2008) invita a pensar críticamente la idealización de una "mujer del Tercer Mundo" genérica. Los feminismos occidentales, sean del Norte Global o los que representan a mujeres blancas de clase media urbana en los paises del Sur, han constituido un discurso efectivo que interpenetra las instituciones estatales y globales y que tiene efectos de colonización al suprimir las heterogeneidades materiales e históricas de las mujeres. Esta visión critica se extiende también para no reproducir la idea de un "hombre genérico del Tercer Mundo", al considerar que la categoria "hombre" no es homogénea, y debe entenderse atravesada por asimetrias de clase, raza, orientación sexual, edad, que distribuyen costos y beneficios de la masculinidad de manera desigual (Connell 2015; Viveros-Vigoya 2018).

Con este escenario, en las secciones siguientes se analizará cómo los estudios globales sobre políticas "hacen género" a través de la elaboración de conceptos, instrumentos e indicadores de evaluación de los impactos de estas políticas en los países de la región, y los efectos de poder producidos.

\section{Autonomía e igualdad en evaluaciones} de la Cepal

La Cepal, creada en 1948, es una de las comisiones regionales de las Naciones Unidas que promueve las relaciones económicas de los paises en el contexto mundial. El Observatorio de Igualdad de Género de América Latina y el Caribe vinculado a esta institución fue creado en 2009 como demanda de la X Conferencia Regional sobre la Mujer de América Latina y el Caribe, en Quito (2007), y promueve estudios de identificación y análisis de las políticas de igualdad de género. Entre sus publicaciones, se eligió analizar Planes de igualdad de género en América Latina y el Caribe: rutas para el desarrollo, basada en un estudio sobre los procesos de diseño de estos Planes desde la década de 1990 en adelante (Cepal 2017).

El propósito del estudio fue evaluar los esfuerzos desplegados por los Estados latinoamericanos y potenciar el trabajo intersectorial para implementar políticas "innovadoras y efectivas" Cepal 2017, 7), que orientan a los paises en el 
alcance del objetivo de promover la igualdad de género de la Agenda 2030 de Desarrollo Sostenible ONU, refrendado en la Estrategia lanzada en la XIII Conferencia Regional sobre la Mujer de América Latina y el Caribe, Montevideo, 2016.

Los conceptos clave manejados por este estudio son igualdad y autonomía. En la introducción son anunciadas dos cuestiones de análisis: si los Planes constituyen "un mapa de ruta hacia la igualdad de género para el Estado en su conjunto o solamente para el mecanismo para el adelanto de las mujeres" (Cepal 2017, 7), y si los contenidos de los Planes reflejan las prioridades del Estado en relación con las desigualdades de género y con los límites en el ejercicio de la autonomía de las mujeres.

En primer lugar, es conceptualizada la igualdad:

Entendida como titularidad de derechos, reafirma el papel del Estado en el logro de umbrales minimos de bienestar para toda la población y su consecución no resta impulso ni recursos al dinamismo económico. Por el contrario, es la clave para una agenda de desarrollo compartida entre los diversos actores. Al ser las desigualdades el resultado de una relación compleja de hechos políticos, sociales, culturales y económicos, las posibilidades para superar esta complejidad exigen politicas integrales y un papel activo del Estado, coherencia entre las políticas económicas y sociales, fortalecimiento de la institucionalidad democrática, superación de la desigualdad territorial y un profundo cambio cultural. (Cepal 2017, 11).

Esta definición refleja una idea universalizante de Estado, responsable de garantizar "umbrales mínimos de bienestar", que parecen corresponder a un techo de la intervención del Estado para el goce efectivo de los derechos de las mujeres. También hay un fuerte vínculo de la igualdad con la idea de "desarrollo", aunque éste no sea definido, pero es un concepto central de la Cepal, principalmente usado para diseminar el formato de politicas focalizadas para el combate a la pobreza y relacionadas con la agenda global de "género y desarrollo" de las décadas de 1980 e 1990. Cabe mencionar que esta agenda fue viabilizada, por ejemplo, para combatir la "feminización de la pobreza", con acciones focalizadas en mujeres pobres urbanas y rurales. Un actor central del proceso fue la Cepal que, desde las décadas de 1950 y 1960, direccionaba la región para fomentar industrialización y substitución de importaciones, encaminando los paises por las "vías de desarrollo" (Escobar 2007).

La publicación de 2017 establece distinciones entre la aplicación del concepto de igualdad de género en tres tipos de políticas públicas, con diferentes impactos de transformación: las políticas de igualdad de oportunidades, con objetivo de incentivar los méritos individuales, pero con poca incidencia en la diminución de la desigualdad; las acciones afirmativas (como los sistemas de cupos electorales) para alcanzar "igualdad sustantiva" enfocadas en la corrección de la desigualdad, con incidencia en la representatividad pero con poco impacto en la reversión de políticas masculinistas; y las políticas de transversalización. Éstas actúan en todo el ciclo de implementación de políticas con el objetivo de redistribución de recursos. Según el estudio, la transversalización se propone un cambio a largo plazo en el orden de género, siendo justamente este tipo de abordaje el que más faltó en los Planes analizados (Cepal 2017).

Cabe anotar que el estudio resalta que la incorporación de la transversalización de género en muchos de los Planes analizados se combina con múltiples discriminaciones que enfrentan las mujeres, como migrantes, indigenas, afrodescendientes, disidentes sexuales, por edad, por discapacidad. También se resalta que el enfoque interseccional está presente sólo en las politicas para las mujeres, y ausente en otro tipo de planes. Interesante anotar también que la interseccionalidad enunciada en el estudio aparece sólo como un dato que los propios Planes traen y no como un indicador de evaluación que la Cepal utilice para pensar en los avances hacia la igualdad de género.

El concepto de autonomía aparece vinculado al "logro de la igualdad", que debe ser "adquirida" de manera individual y colectiva. En el caso de las mujeres, la autonomía está relacionada a los espacios de poder que puedan ocupar y asi lograr individualmente y como grupo social un grado mayor de libertad para sus acciones. Parece haber una brecha conceptual y práctica entre la dimensión micro-social de la autonomía 
(ubicada entre "la" mujer como individualidad y "las" mujeres como grupo social) y la dimensión macro-social de la igualdad.

Para la construcción de indicadores, el estudio define tres grandes grupos de autonomias: física, en la que se examinan avances en relación con la violencia contra las mujeres y la salud; en la toma de decisiones, sobre la presencia de mujeres en el ejercicio del poder público; y económica, sobre el control de las mujeres de recursos monetarios y de tiempo, incorporación al empleo y situaciones de pobreza. Un cuarto grupo abarca la interrelación de autonomías a través de indicadores de educación, entre otros.

Es a través de estos indicadores que se analizan objetivos y metas de los Planes y la realización de políticas públicas, con el propósito de "hacer una lectura de los avances y rezagos en estas dimensiones de la desigualdad de género" (Cepal 2017, 72). El análisis de las autonomías se da a partir de un grupo acotado de indicadores estratégicos consensuados por los gobiernos, y de repositorios legislativos. Analizaremos la autonomía física, que es la que encadena género con indicadores de salud.

Los indicadores de autonomía física examinados en el estudio son: Mortalidad materna; Maternidad en adolescentes (de 15 a 19 años); Demanda insatisfecha de planificación familiar (brecha entre intenciones reproductivas de la mujer y su comportamiento anticonceptivo); Feminicidio; 4 Muerte de mujeres ocasionada por su pareja o expareja intima; y Muerte de mujeres ocasionada por su pareja o expareja intima. Para todos estos indicadores son detalladas variables y fuente de los datos.

Los tres primeros indicadores se refieren a problemáticas sanitarias que según el estudio fueron históricamente resaltadas en los Planes. La mortalidad materna es considerada como una preocupación mundial y regional desde la década de 1980, abordando las causas prevenibles a través de acciones orientadas por agencias internacionales. Las otras dos, el embarazo no planificado y el embarazo en la adolescencia, están directamente relacionadas con la discusión de autonomía, especialmente autonomía reproductiva, que la Cepal vincula a su incidencia en la autonomía económica. En este ámbito, se evalúa que "las medidas siempre parecen insuficientes y la maternidad en edades tempranas, que trunca las posibilidades de estudio y de futura inserción laboral de las mujeres, sigue impactando en especial a las mujeres más pobres" (Cepal 2017, 33).

Hay aqui un punto clave que remite a las ideas de "género y desarrollo" y a la racionalidad neoliberal citada. Parece naturalizada la necesidad de regular la reproducción de las mujeres pobres, sea para disminuir el número de hijos, sea para evitar la maternidad en edades tempranas, ya que tendría un impacto económico (naturalizado e individualizado). Éste ha sido el centro de las orientaciones de las políticas de combate a la pobreza en los países del Sur desde actores globales, junto a medidas de ajuste estructural. En ese sentido, los países son evaluados de acuerdo a si incentivan o no esa regulación, que debe reflejarse en las responsabilidades individuales de las mujeres. En ningún momento se menciona el enfrentamiento de las desigualdades y la necesidad de medidas robustas de justicia redistributiva y de reconocimiento que permitan el acceso de esas mujeres a derechos básicos, como educación y trabajo, y/o que los costos sociales de la maternidad sean amparados en la esfera pública para tener una posibilidad real de elegir.

Estas evaluaciones de avances de políticas parecen recurrir a un mediador moral externo a las realidades de esos grupos, basado en lo que debería ser una situación idealizada de derechos individuales y sociales garantizados para las mujeres sujetas de estas politicas y naturalizando una forma de ser mujer y madre, entre otras, desde los discursos institucionales del Estado. ${ }^{5}$

\footnotetext{
4 Según informe de ONU Mujeres Análisis sobre legislación de femicidio/feminicidio en América Latina y el Caribe e Insumos para una Ley Modelo (Deus y Gonzalez 2018), todos los paises de América Latina, excepto Cuba y Haiti, han aprobado leyes que penalizan el femicidio/ feminicidio. Sin embargo, cuando fue realizado el estudio de la Cepal es probable que varios de estos países no hubieran implementado esta ley.

5 Para ampliar estas discusiones a través de estudios etnográficos, recomendamos Robles (2015) y Montiel y López (2020), entre otros.
} 
Es producida una "mujer del Tercer Mundo" genérica que, como sugiere Mohanty $(2008,126)$, "Lleva una vida esencialmente truncada debido a su género femenino" y a su condición de "ignorante, pobre, sin educación, limitada por las tradiciones, doméstica, restringida a la familia, victima", contrapuesta a "la autorrepresentación (implícita) de la mujer occidental como educada, moderna, con el control de su cuerpo y su sexualidad y con la libertad de tomar sus propias decisiones".

Sobre autonomía reproductiva, y reflexionando sobre el contexto violento de esterilización masiva de mujeres negras, indigenas y pobres y las denuncias públicas realizadas por movimientos que representan a esas mujeres, ${ }^{6}$ Roland (1995) abre las siguientes preguntas: ¿cómo viabilizar el lema feminista "nuestro cuerpo nos pertenece" cuando no se producen condiciones para la autonomía de la mayoría de las mujeres? ¿Cómo pensar la autonomía de las mujeres en relación a su reproducción en contextos en que la experiencia social del cuerpo se vive a través de profundas desigualdades?

La supresión de heterogeneidad en la construcción de una idea de autonomía alejada de contextos y dinámicas de vida de la mayoría de las mujeres latinoamericanas, es uno de los puntos a resaltar de las operaciones discursivas de la Cepal. Dicha supresión es reforzada en la construcción de instrumentos cuantitativos para evaluar las políticas de igualdad de género.

Cuando se explican los indicadores de embarazo en la adolescencia o de demandas insatisfechas de planificación familiar no son informados, por ejemplo, datos sobre anticoncepción entre los hombres. Tampoco son incluidas caracteristicas sociodemográficas de las mujeres (clase, raza etc.). Esto sucede también con los otros indicadores.

Si pensamos en la violencia contra las mujeres (feminicidios y muertes ocasionadas por su pareja), podría ser comparado las muertes por causa violenta de mujeres y hombres, y cuáles son las mujeres y los hombres que mueren más, considerando raza, clase, disidencias sexuales y de género. Es notorio, por ejemplo, que personas LGBT+ (lesbianas, gays, bisexuales, travestis, trans y otras expresiones de género no binarias) no aparecen contempladas en estos indicadores, pese a que la producción académica sobre género llama la atención de la relevancia de desconstruir los binarismos, referentes a sexo, género o raza (Gomes 2018).

En relación con las políticas de género y desarrollo, como alerta Escobar (2007, 303), "[e]l uso de procedimientos y estadísticas estandarizados hace inevitable cierta desaparición de la experiencia de la mujer". Así, este análisis muestra que la Cepal en su estudio refuerza una perspectiva categorial, focalizada en una mujer genérica, en la cual la interseccionalidad y las relaciones de género no aparecen ni en la producción de indicadores, ni como conceptos y ejes de análisis.

\section{La equidad de género en las producciones de la OPS}

La OPS es una organización internacional especializada en salud de las Américas, cuyo énfasis es el mejoramiento y la protección del derecho a la salud. Al revisar su producción, seleccionamos tres publicaciones del 2019, que plantean una propuesta de análisis de género y salud. Según consta en los textos, los estudios fueron elaborados para subsidiar la implementación de la Estrategia para el Acceso Universal a la Salud y la Cobertura Universal de Salud de la Organización Mundial de la Salud ${ }^{7}$ en materia del logro de la igualdad de género. El análisis se pregunta de qué manera complejizan la propuesta conceptual y analítica de la Cepal para abordar y "hacer género", así como cuáles son sus límites.

Comenzamos con la publicación Marco conceptual e indicadores para monitorear la igualdad de género en la salud en las Américas (OPS 2019a), cuyo objetivo fue proponer un marco y un conjunto actualizado de indicadores básicos para monitorear el avance hacia la igualdad de género en la 
salud en los países de la región. Se considera que existe una situación persistente de inequidad en materia de salud entre mujeres, hombres, niñas y niños, abarcando también las minorias sexuales, las personas que son objeto de discriminación étnica-racial y los adultos mayores. Este texto, así como otros de la OPS, distingue los conceptos de igualdad y de equidad de la siguiente manera:

La igualdad describe un estado de homogeneidad o comparabilidad entre dos o más grupos o personas en el goce de las mismas oportunidades de acceso y control de recursos sociales, económicos y políticos. La equidad, sin embargo, implica justicia y es relativa, basada en las diferentes necesidades, preferencias e intereses de individuos o grupos sociales y se logra cuando se eliminan las diferencias evitables, sistemáticas y sistémicas. [...] La equidad es el medio para lograr la igualdad. (OPS 2019a, 4).

Este concepto está basado en la definición de Whitehead, que, en 1992, planteó las inequidades en salud como "diferencias innecesarias, evitables, injustas e injustificadas, y así, posicionó la equidad en salud como un tema de justicia social" (OPS 2019a, 3). De esta manera, la equidad de género implica una "distribución justa de los beneficios, el poder, los recursos y las responsabilidades entre las mujeres y los hombres", reconociendo las diferencias "en cuanto a las necesidades, el acceso y el control de los recursos de salud, y su objetivo es abordarlas para corregir desequilibrios entre hombres y mujeres" (OPS 2019a, 9). Las estrategias de equidad de género se utilizan para lograr la igualdad en la atención a la salud, las condiciones de vida, el trabajo sanitario, reconociendo la heterogeneidad de hombres y mujeres por su situación socioeconómica, violencia, empleo, origen étnico/racial y edad.

Se puede decir que este concepto de equidad se tornó hegemónico en los discursos de los actores globales, provocando una tensión con la conceptualización vinculada a nociones de justicia social, que tiene su historia en las luchas latinoamericanas por el derecho a la salud, entrelazadas con las movilizaciones políticas para la democratización y la paz. El concepto de equidad es clave en el campo político-conceptual de la salud pública contemporánea y principalmente de la salud colectiva y la medicina social (Silva y Almeida-Filho 2009).

Según Basile (2020), la equidad es una de las nuevas terminologías e intervenciones de las tecno-burocracias de organismos internacionales para relanzar las reformas de mercados (des) regulados en los sistemas de salud, con expansión de las aseguradoras financieras y el sector privado a través de la Cobertura Universal de Salud, profundizando la lógica neoliberal.

Planteamos que se usa el concepto despolitizado, dado que no se reconoce que las inequidades son producto de relaciones de poder asimétricas entre posiciones desiguales de grupos sociales y que, en su raíz teórica y ético-política implica acciones de justicia distributiva concreta (Silva y Almeida-Filho 2009), aplicadas a inequidades históricas que demandan cambios urgentes; para las mujeres diversas la superación de inequidades demanda además reconocimiento. Esta despolitización se relaciona con paulatina privatización de los sistemas de seguridad social (incluyendo el sector salud) de la región, promovida por las políticas neoliberales (Fleury 2018).

Sobre el concepto de género usado por OPS, se identifica el camino hacia una perspectiva relacional, y así la apertura a otras posibilidades de captar la heterogeneidad a través del abordaje interseccional. Si para Cepal, la interseccionalidad era un "dato" que surgía de la realidad, para la OPS aparece como un abordaje para trabajar la heterogeneidad de condiciones de vida y experiencias sociales de hombres y mujeres.

Las publicaciones de OPS manejan una definición de género como "conjunto de atributos (símbolos y normas) y roles (identidades) de construcción histórica y cultural que se preservan a través de actos en la organización social cotidiana", permitiendo entender "cómo se convierten en desigualdad las diferencias entre hombres y mujeres" (OPS 2019b, 3).

Para operacionalizar esta concepción, fueron construidos indicadores que ayudan a "describir la situación y las experiencias de mujeres, hombres y minorias sexuales, asi como la influencia 
de otras variables, como el lugar de residencia, la edad, la orientación sexual, la etnicidad, los ingresos personales o familiares." (OPS 2019a, 36). Los indicadores sensibles al género proporcionan información que puede servir para detectar situaciones de desigualdades entre los géneros en los ámbitos familiares, comunitarios y en las estructuras sociales y politicas, y evaluar si éstas "impiden injustamente que algunas mujeres o algunos hombres tengan participación plena en la sociedad o logren realizar su potencial total de salud", así como "monitorear qué grupos de población de hombres y niños se encuentran en una situación de vulnerabilidad". (OPS 2019a, 37).

Los indicadores construidos para este fin son: 1) Indicadores género-sensibles para mortalidad y morbilidad; 2) Necesidades de cada género en salud reproductiva, desglosados por edad e incorporando la salud sexual; 3) Violencia sufrida por mujeres y hombres, y existencia de estructuras estatales de protección; 4) Indicadores de trabajo no remunerado; 5) Financiamiento público del sistema de salud; 6) Degradación ambiental; 7) Cambios en las condiciones climáticas; 8) Participación y compensación equitativa de mujeres y hombres en la fuerza laboral; 9) Igualdad de género en la formulación de políticas y gobernanza. Interesante anotar que la publicación resalta la necesidad del uso de instrumentos cualitativos adicionales para conocer profundamente las diferencias, siendo los estudios en escala micro una manera más pertinente para analizar las desigualdades en salud de grupos minoritarios y conocer contextos de vida localizados de mujeres y hombres.

Se observa un avance en la consideración de una perspectiva relacional en la producción de indicadores. La mención a métodos cualitativos abre la posibilidad de analizar dinámicas más que categorias. Esta posibilidad es ampliada en la obra Transversalización de género en salud: avances y desafios en la Región de las Américas, que analiza los avances y desafíos pendientes de la implementación de esta perspectiva (OPS 2019c).

La publicación destaca que los sistemas de información de la región siguen trabajando con una definición binaria de género y no se detectó publicaciones que documenten el uso de modelos que amplien el binarismo. ${ }^{8}$ Se continúa desagregando las informaciones por sexo, lo que no es suficiente para analizar las desigualdades de género en salud, que necesita de otras variables de los determinantes sociales y de registro de datos sobre LGBT+, que contemplen pluralidad de género y prácticas sexuales.

Sobre monitoreo y evaluación de políticas, se visibilizó la demanda por sistematización de experiencias y la importancia de incorporar instrumentos cualitativos que permitan "retratar mejor el impacto de las relaciones de género en la salud y el bienestar" (OPS 2019c, xii), así como "evaluar los cambios en la distribución de poder y recursos por razón de género, los cuales representan el núcleo del cambio social buscado" (OPS 2019c, 33). En el plano teórico-metodológico, se sugirió la "triangulación con análisis cualitativos para avanzar en la comprensión de la experiencia vivida por las mujeres y los hombres, utilizando enfoques participativos que sean sensibles a la distribución de poder en los procesos a explicar y en su análisis" (OPS 2019c, y42).

Resaltamos que en las publicaciones analizadas, aunque se enuncie la equidad de una manera abstracta y se refuerce una "versión neoliberal" del concepto, el término parece abrir la posibilidad de analizar en profundidad las políticas de género, sea considerando las dinámicas de desigualdades, sea en la evaluación crítica de producción de indicadores. Esto se articula con perspectivas de género relacional e interseccional, lo que permite una construcción de género diferente de la realizada por Cepal, creando instrumentos de evaluación de las políticas locales que puedan ser más reflexivos en relación a sus impactos.

\section{Conclusiones}

En este artículo, presentamos un análisis discursivo de las políticas públicas que articulan género y salud en América Latina, abordando

8 Canadá es el único país de las Américas que institucionalizó un modelo de análisis (Genderbased Analysis Plus - GBA+) que amplía las categorizaciones y adopta una definición no binaria de género (OPS 2019c). 
cómo los estudios y evaluaciones globales "hacen género" de una manera particular. Notamos diferencias en los modos de "hacer género" en los discursos producidos por dos actores globales (Cepal y OPS), relacionados con los conceptos, indicadores e instrumentos de evaluación de políticas que proponen en sus estudios. Por un lado, observamos una operación discursiva de supresión de heterogeneidad a partir de la idea de autonomía de Cepal. Por otro, la OPS se mostró más plural con sus construcciones relacionales de género que se orientaban por nociones como equidad y contemplaban experiencias de hombres y mujeres en su diseño. A pesar de ello, destacamos que el alineamiento de ambos organismos a las políticas neoliberales no permite visualizar instrumentos de justicia social más robustos propuestos en estas evaluaciones.

Esta escritura finaliza en contexto de pandemia, en el que las desigualdades históricas se potencializan y muestran que la equidad pretendida a través de políticas públicas no se concretizó, pues las estructuras desiguales de nuestras sociedades siguen cristalizadas. Incluso porque los actores globales y sus nociones de igualdad, autonomía y equidad siguen reproduciendo dispositivos descontextualizados, despolitizados y de supresión de heterogeneidad, siendo que estos actores continúan sin posicionarse como parte de la producción de desigualdades.

Frente a este panorama, y a partir de estos resultados, recordamos el hecho de que dispositivos, como los MAM, por ejemplo, fueron potentes como parte de la respuesta a demandas de los movimientos feministas desde finales del siglo 20. Sin embargo, fueron limitados en la perspectiva de lograr una incidencia profunda en la producción de justicia social con mecanismos de reconocimiento y redistribución que provoquen transformaciones profundas en el orden de género, sea por no conectar esas mujeres con las dinámicas de género (al no considerar, por ejemplo, a los hombres en las políticas), o por naturalizar una "mujer genérica del Tercer Mundo" (y un hombre genérico) sin conexión con otras dinámicas de dominación de clase, raza, sexualidad, y con el orden global, que reproducen la colonialidad en nuestras sociedades. Por ello, interpelamos por la necesidad de elaborar instrumentos de equidad desde el Sur, para incidir en el orden de género y la colonialidad imperante en nuestra región, buscando salidas más alineadas con concepciones de justicia social construidas por las luchas sociales latinoamericanas.

\section{Referencias}

Basile, Gonzalo. 2020. Repensar y descolonizar la teoria y políticas sobre sistemas de salud en América Latina. Cuadernos del Pensamiento Crítico Latinoamericano, 73: 1-6. https://www.clacso.org/wp-content/ uploads/2020/05/Cuaderno-PLC-N73-Abril_2020.pdf.

Berardi, Leda, comp. 2003. Análisis Critico del Discurso. Perspectivas latinoamericanas. Santiago: Frasis Editores.

Comisión Económica para América Latina y el Caribe (Cepal). 2017. Planes de igualdad de género en América Latina y el Caribe. Mapas de ruta para el desarrollo. Observatorio de Igualdad de Género en América Latina y el Caribe. Estudios $n^{\circ}$ 1. Santiago de Chile: Cepal. https://www.cepal.org/sites/default/files/events/files/ planes_de_igualdad_de_genero_en_america_latina_y_ el_caribe._mapas_de_ruta_para_el_desarrollo.pdf.

Connell, Raewyn. 2013. Género, salud y teoria: conceptualizando el tema en perspectiva mundial y local. Nómadas 39: 63-77. https://www.redalyc.org/ pdf/1051/105129195005.pdf.

Connell, Raewyn. 2015. El género en serio. Cambio global, vida personal, luchas sociales. México DF: Universidad Nacional Autónoma de México.

Damasco, Mariana, Marcos Maio y Simone Monteiro. 2012. Feminismo negro: raça, identidade e saúde reprodutiva no Brasil (1975-1993). Revista Estudos Feministas 20 (1): 133-151. https://doi.org/10.1590/S0104026X2012000100008.

Del Río Fortuna, Cynthia. 2013. Población, reproducción y derechos: un estudio comparativo de la politica de acceso a la anticoncepción quirúrgica femenina en Brasil y Argentina. Sexualidad, Salud y Sociedad 13: 9-42. https://doi.org/10.1590/S1984-64872013000100002.

Deus, Alicia y Diana Gonzalez. 2018. Análisis sobre legislación de femicidio/feminicidio en América Latina y el Caribe e Insumos para una Ley Modelo. ONU Mujeres. Panamá: Casa de las Naciones Unidas.

Escobar, Arturo. 2007. La invención del tercer mundo. Construcción y deconstrucción del desarrollo. Caracas: Fundación Editorial El Perro y La Rana.

Fahra, Martha. 2004. Gênero e políticas públicas. Revista Estudos Feministas 12 (1): 47-71. https://doi.org/10.15.90/ S0104-026X2004000100004.

Fleury, Sonia. 2018. Capitalismo, democracia, cidadania - contradições e insurgências. Saúde em Debate 42: 108-124. https://doi.org/10.1590/0103-11042018s309. 
Gomes, Camilla. 2018. Gênero como categoria de análise decolonial. Civitas - Revista de Ciências Sociais 18 (1): 65-82. https://doi.org/10.15448/19847289.2018.1.28209.

Guzmán, Virginia y Claudia Bonan Jannotti. 2008. La institucionalización del tema de la equidad de género y la modernización del Estado en América Latina. Aportes para el debate 25: 103-114. https:// www.asociacionag. org.ar/pdfaportes/25/07.pdf.

Guzmán, Virginia y Sonia Montaño. 2012. Politicas públicas e institucionalidad de género en América Latina (1985-2010). Santiago de Chile: Cepal. https://repositorio.cepal.org/bitstream/handle/11362/5847/1/ S1200803_es.pdf.

Lugones, María. 2008. Colonialidad y género. Tabula Rasa 9: 73-101. https://doi.org/10.25058/20112742.340.

Mohanty, Chandra. 2008. Bajo los ojos de Occidente. Academia feminista y discursos coloniales. In Descolonizando el Feminismo: teorias y prácticas desde los márgenes, compiladoras Liliana Suárez Navaz y Aida Hernández, 117-163. Madrid: Cátedra.

Montiel, Carolina y Laura López. 2020. Trajetórias reprodutivas femininas e produção do cuidado em saúde orientado às gestantes na cidade de São Leopoldo - RS: um olhar interseccional. Revista Gênero 20 (2): 300-322. https://doi.org/10.22409/rg.v20i2.44580.

Organización Panamericana de la Salud (OPS). 2019a. Marco conceptual e indicadores para monitorear la igualdad de género en la salud en las Américas. Washington: OPS. https://iris.paho.org/handle/10665.2/51785.

Organización Panamericana de la Salud (OPS). $2019 \mathrm{~b}$. Masculinidades y salud en la Región de las Américas. Washington: OPS. https://iris.paho.org/handle/10665.2/51667.

Organización Panamericana de la Salud (OPS). 2019c. Transversalización de género en salud: avances y desafios en la Región de las Américas. Washington: OPS. https://iris.paho.org/handle/10665.2/51809.

Robles, Alfonsina. 2015. Regulações do corpo e da parentalidade durante o pré-natal em mulheres jovens de camadas populares. Civitas - Revista de Ciências Sociais 15 (2): 190-213. https://doi.org/10.15448/19847289.2015.2.17985.

Roland, Edna. 1995. Direitos reprodutivos e racismo no Brasil. Revista Estudos Feministas 3 (2): 506-514. https:// doi.org/10.15.90/\%25x.

Silva, Ligia Maria y Naomar Almerida-Filho. 2009. Equidade em saúde: uma análise crítica de conceitos. Cadernos de Saúde Pública 25 (2): 217-226. http://dx.doi. org/10.1590/S0102-311X2009001400004.

Tajer, Débora. 2012. Construyendo una agenda de género en las políticas públicas en salud. In Género y Salud. Las politicas en acción, 14-20. Buenos Aires: Lugar Editorial.
Vargas, Virginia. 2003. Los feminismos latinoamericanos y sus disputas por una globalización alternativa. In Politicas de identidades y diferencias sociales en tiempos de globalización, compilado y coordinado por Daniel Mato, 193-217. Caracas: Faces - UCV.

Vianna, Adriana y Laura Lowenkron. 2017. O duplo fazer do gênero e do Estado: interconexões, materialidades e linguagens. Cadernos Pagu 51: e175101. https://doi. org/10.1590/18094449201700510001.

Viveros Vigoya, Mara. 2018. As cores da masculinidade. Experiências interseccionais e práticas de poder na Nossa América. Rio de Janeiro: Papeis Selvagens

\section{Laura Cecilia López}

Doctora y magister en Antropologia Social de la Universidade Federal do Rio Grande do Sul (Ufrgs), en Porto Alegre, RS, Brasil. Antropóloga de la Universidad de Buenos Aires (UBA), en Buenos Aires, Argentina. Profesora en los Programas de Posgrado en Salud Colectiva y en Ciencias Sociales de la Universidade do Vale do Rio dos Sinos (Unisinos), en São Leopoldo, RS, Brasil.

\section{Luanda Rejane Soares Sito}

Doctora y magister en Lingüistica Aplicada por el Instituto de Estudios del Lenguaje, de la Universidade Estadual de Campinas (Unicamp), en Campinas, SP, Brasil. Licenciada en Letras de la Universidade Federal do Rio Grande do Sul (Ufrgs), en Porto Alegre, Brasil. Profesora en la Facultad de Educación de la Universidad de Antioquia (UdeA), en Medellín, Colombia.

\section{Yadira Eugenia Borrero-Ramírez}

Doctora en Salud Pública de la Universidad de Antioquia (UdeA), en Medellin, Colombia y magister en Sociología de la Universidad del Valle (Univalle), en Cali, Colombia. Médica de la Universidad Nacional de Colombia (Unal), en Bogotá, Colombia. Profesora en la Facultad Nacional de Salud Pública de la Universidad de Antioquia (UdeA), en Medellín, Colombia.

Os textos deste artigo foram conferidos pela Poá Comunicação e submetidos para validação do(s) autor(es) antes da publicação. 